\title{
Radiometric characterization of hyperspectral imagers using multispectral sensors
}

\author{
Joel McCorkel, Kurt Thome, Nathan Leisso, Nikolaus Anderson and Jeff Czapla-Myers \\ Remote Sensing Group, College of Optical Sciences, University of Arizona, Tucson AZ 85721
}

\begin{abstract}
The Remote Sensing Group (RSG) at the University of Arizona has a long history of using ground-based test sites for the calibration of airborne and satellite based sensors. Often, ground-truth measurements at these tests sites are not always successful due to weather and funding availability. Therefore, RSG has also employed automated ground instrument approaches and cross-calibration methods to verify the radiometric calibration of a sensor. The goal in the cross-calibration method is to transfer the calibration of a well-known sensor to that of a different sensor. This work studies the feasibility of determining the radiometric calibration of a hyperspectral imager using multispectral imagery. The work relies on the Moderate Resolution Imaging Spectroradiometer (MODIS) as a reference for the hyperspectral sensor Hyperion. Test sites used for comparisons are Railroad Valley in Nevada and a portion of the Libyan Desert in North Africa. Hyperion bands are compared to MODIS by band averaging Hyperion's high spectral resolution data with the relative spectral response of MODIS. The results compare cross-calibration scenarios that differ in image acquisition coincidence, test site used for the calibration, and reference sensor. Cross-calibration results are presented that show agreement between the use of coincident and non-coincident image pairs within $2 \%$ in most bands as well as similar agreement between results that employ the different MODIS sensors as a reference.
\end{abstract}

Keywords: cross calibration, hyperspectral, desert sites

\section{INTRODUCTION}

There are dozens of earth observing satellite sensors currently on orbit for the purpose of land- and ocean-based remote sensing. Data reported by these sensors provide a basis for a wide range of environmental studies. It is critical that sensors remain calibrated in order to achieve synergy among coexisting sensors and legacy among generations of sensors. There are many available methods of radiometric calibration of satellite sensors including prelaunch, on-board lamp, lunar, or solar illumination, and methods that use terrestrial scenes acquired on-orbit. The RSG has historically employed the reflectance-based method of vicarious calibration for a large array of sensors. The basic method primarily relies on manned, in situ measurements of a test site's surface and atmospheric conditions close in time to when the sensor of interest views that site ${ }^{1}$. Recent developments by the RSG allow these measurements to be autonomous without the requirement for on-site personnel ${ }^{2}$.

One critical goal of the in-flight radiometric calibration of a sensor is to determine that sensor's calibration as soon as possible after launch. The rigors of launch often invalidate the results of the preflight calibration. On-board calibration hardware has also been shown to change in going from the laboratory to the space environment therefore additional validation is preferred ${ }^{3}$. Approaches relying on in-situ measurements have been shown to provide this validation but often prove ineffective for rapid evaluation of sensor calibration. Poor weather conditions, lack of personnel, or limited financial resources can prevent the number of usable data collections and limit the frequency of the in-situ data. Crosscalibration methods provide an opportunity for vicarious calibration at a higher temporal frequency than the in-situ approaches.

The two sensors involved in a cross-calibration each have their own orbit and their imagery will be acquired with unique viewing and solar geometries. The ideal case for cross-calibration is when the two sensors share the same orbit or even the same spacecraft. For example, before arriving to its destination orbit, Landsat 7 was placed in a tandem orbit with Landsat 5 allowing for the cross calibration of the Landsat 7 Enhanced Thematic Mapper Plus (ETM + ) and Landsat 5 Thematic Mapper (TM) to establish calibration continuity, ${ }^{4,5}$. Moving away from this ideal is the case where the two sensors acquire imagery at different times such as attempting the comparison between ETM+ and Terra MODIS, which lags ETM + by approximately 40 minutes $^{6}$. These and other, less ideal, scenarios for cross-calibration of two satellite sensors are listed below: 


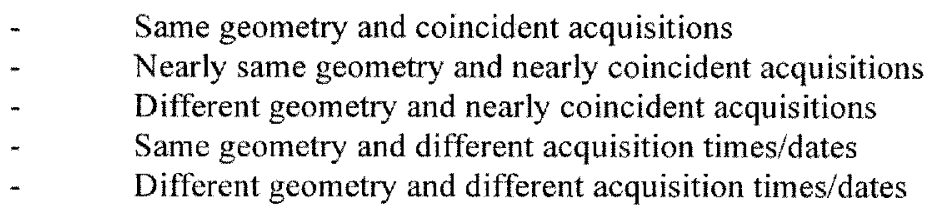

In this paper, geometry refers to the viewing zenith angle and solar zenith angle at the time of image acquisition. As mentioned, the most accurate case is when two sensors view the same area at the same time with the same geometry. Any difference in acquisition time or date increases uncertainties due to changes in surface and atmospheric conditions affecting the radiance at the two sensors. The effect of the atmosphere and surface are further exacerbated when the two sensors view the same area at different view angles.

This work studies cross calibration results for the two of the above mentioned scenarios: 1) the sensors have the same geometry and nearly coincident image acquisitions; and 2) the sensors have the same geometry but different acquisition dates. Results for both of these scenarios are presented for the comparison between Hyperion and MODIS (Terra and Aqua). Studying both scenarios with the same sensor pair is possible because Hyperion's orbit preceded MODIS by 40 minutes early in its mission but experienced an orbit change in mid-2005, after which only non-coincident comparisons are possible.

This paper gives a brief overview of the sensors used for the cross-calibration and then discusses the test sites used in the comparisons. Methodology is presented on how the comparisons were made for the two different cross-calibration scenarios. The results section looks at the agreement between comparisons made with the nearly coincident datasets and comparisons made with datasets that have been acquired on different dates. Also shown is the agreement between results obtained with a Hyperion-Terra MODIS comparison and a Hyperion-Aqua MODIS comparison. To provide a comparison between Hyperion and MODIS independent of the method described in this work, the cross-calibration results are compared with results obtained from the reflectance-based approach of vicarious calibration, which takes advantage of ground-truth measurements.

\section{SENSORS}

Multispectral sensors tend to have wider swath widths and more frequent acquisitions of any given site relative to a hyperspectral imager. In addition, hyperspectral sensors are usually tasked by an operator, meaning that even when the sensor is over a desired area there may be no image acquisition. The outcome is that access to a multispectral, wide swath image of a desired test site will not mean access to a similar hyperspectral image. On the other hand, there is a high probability of having an overlapping multispectral scene for any given hyperspectral acquisition.

There are many multispectral sensors in orbit that have trusted absolute radiometric calibrations. This work uses data from the MODIS sensors on the Terra and Aqua platforms due to their remarkably accurate radiometry calibration ${ }^{7,8}$. Frequent revisit times of every other day at the equator and daily coverage at mid-latitudes is essential for this work and provided by the MODIS sensors. Terra was launched in December 1999 with a 10:30 am equatorial crossing time and Aqua was launched in May 2002 with a $1: 30$ pm equatorial crossing time. Terra is commonly known as the 'AM' satellite and Aqua, the 'PM' satellite due to their equatorial crossing times. MODIS has spatial resolutions of 250,500 , and $1000 \mathrm{~m}$ depending on the spectral band covering a swath width of $2330 \mathrm{~km}$. The MODIS sensors have a total of 36 bands, 19 of which are within the solar reflective region of the electromagnetic spectrum.

Hyperion is the hyperspectral imager selected for this work. One advantage to using the Hyperion sensor here is RSG's history of calibrating it with the reflectance-based approach providing results that can be compared with other studies. Hyperion is onboard the EO-1 spacecraft launched in November 2000. It has 198 processed spectral bands in the 0.4-2.5 $\mu \mathrm{m}$ range with nominal bandwidths of $10-11 \mathrm{~nm}$ and about $10 \mathrm{~nm}$ between band centers ${ }^{2}$. The imager has a ground resolution of $30 \mathrm{~m}$ covering a swath width of $7.7 \mathrm{~km}$.

The reflectance-based calibration results of Hyperion uses imagery that is processed to level $1 \mathrm{R}$. Level 1T data are used for this cross-calibration study. Level $1 \mathrm{R}$ could not be obtained for this study. Level $1 \mathrm{~T}$ data are freely available on the internet and comes with geolocation data. For comparing the results from the reflectance-based approach and the crosscalibration study in this work, it is important that the radiometric scale between the two different levels be known. The solid line in Figure 1 shows the difference between level $1 \mathrm{R}$ and level $1 \mathrm{~T}$ radiances averaged over an area of $1 \mathrm{~km} \times 1$ $\mathrm{km}$ and five different acquisitions at Railroad Valley. The positive vertical axis represents a higher radiance reported in 
level IR. The dashed lines represent plus and minus one standard deviation about the average of the five scenes. With the largest difference approaching $1 \%$ in the shortwave infrared, comparisons between the reflectance-based approach and cross-calibration study are directly compared.

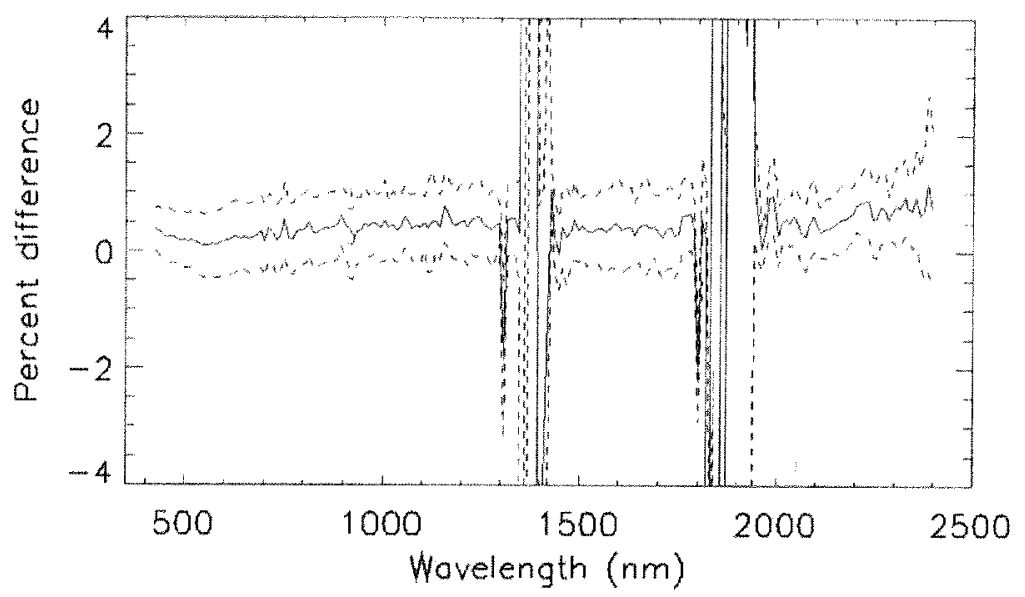

Figure 1. The percent difference curve (solid line) shows a comparison of Hyperion L1R and L1T imagery of a $1 \mathrm{~km} \times 1 \mathrm{~km}$ area of the Railroad Valley test site averaged for five image pairs. The dashed lines represent plus and minus one standard deviation about the average.

\section{TEST SITES}

The ideal test site for reflectance-based calibration has many characteristics. These include a relatively high surface reflectance, and surface properties that are spatially uniform, temporally invariant and near-lambertian surface. It is also desirable that the site be at a high altitude; cover a large area; be an arid region to limit water vapor; and must be accessible ${ }^{10}$. Many of these same properties are needed for accurate cross-calibration as well and the RSG sites serve as the starting point for this work. One site evaluated extensively by RSG is Railroad Valley Playa in Nevada that has been found to satisfy many of the above criteria ${ }^{10}$. RSG has a reasonably large archive of ancillary data and imagery from many sensors at Railroad Valley. Railroad Valley is located at approximately $38.5 \mathrm{~N}, 115.7 \mathrm{~W}$ and is accessible via a common US highway. It offers a relatively high reflectance across the visible and short wave infrared spectrum, shortterm stability, frequently cloud-free skies, and is easily locatable in imagery. It is about $10 \times 15 \mathrm{~km}^{2}$ in area and at 1430 m altitude.

A one-kilometer square area of Railroad Valley that has been used by RSG for the reflectance-based calibration of MODIS is used in this work ${ }^{11}$. A typical reflectance spectrum obtained from in-situ measurements of the area is shown in Figure 2. This area represents a single pixel of the low-resolution bands of MODIS depicted in Figure 3. 


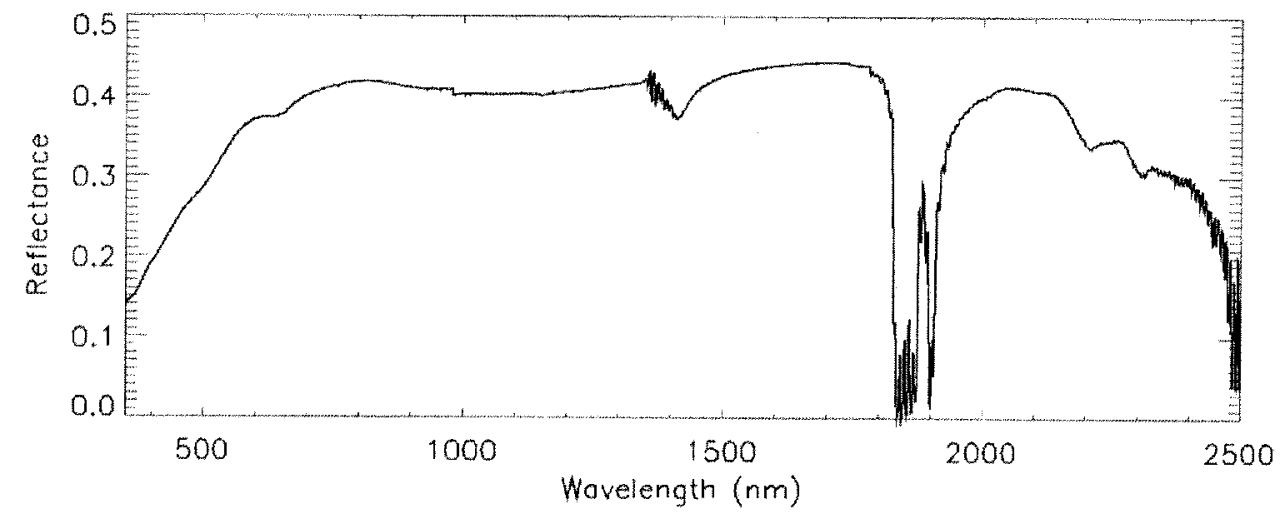

Figure 2. Reflectance of the Railroad Valley test site according to in situ measurements on 13 July 2002.

One criterion listed above not required for cross-calibration is accessibility. Work has shown that portions of the Saharan desert are suitable invariant sites and also meet many of the characteristics listed for an ideal site, especially that of minimal cloud cover and precipitation ${ }^{12}$. Previous work that utilizes the Saharan desert includes temporal trending of sensor calibration ${ }^{13,14}$, sensor intercomparisons ${ }^{15,16,17}$, flat fielding wide field of view sensors by characterizing several sites $^{18}$, and comparison of aerosol optical depth products over desert sites ${ }^{19}$. For this work, a Saharan test site offers the opportunity to utilize a site much larger than Railroad Valley and evaluate the accuracy of using a site for which groundbased data are not available.

The selection of test site area within the Saharan desert is based on the availability of high temporal frequency Hyperion imagery that was readily found in seemingly uniform areas. This search led to scenes in Libya, more specifically in World Reference System (WRS) path 181, row 40, in which nearly 200 scenes have been acquired from 2004 to present. In order to compare consistent portions of the Libyan surface, an area is selected that is slightly narrower than Hyperion's swath width and long as possible while keeping a large buffer away from less uniform areas. Initial judgment of selecting this area was done manually by stretching scenes from several dates and selecting an area that had minimal change spatially and temporally. The resulting area is shown as the highlighted rectangle in Figure 4 and is defined by these coordinates: [29.1056, 23.8091], [29.0958, 23.8687], [28.8180, 23.7956], and [28.8281, 23.7348]. This represents an area of approximately $6 \mathrm{~km} \times 31 \mathrm{~km}$ or about 210,000 Hyperion pixels and almost 200 MODIS pixels. This area is fully contained within the 50 Libyan Hyperion scenes processed in this work despite random longitudinal shifts of spatial coverage.

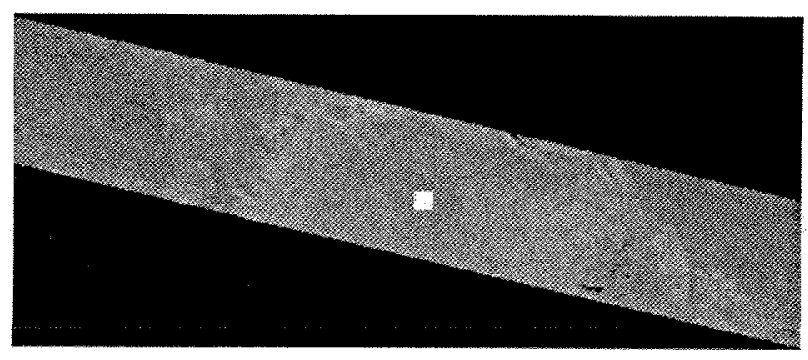

Figure 3. A portion of a typical Hyperion scene of Railroad Valley in WRS path 40 row 33 . The highlighted square in the middle of the playa represents the area used for comparisons.

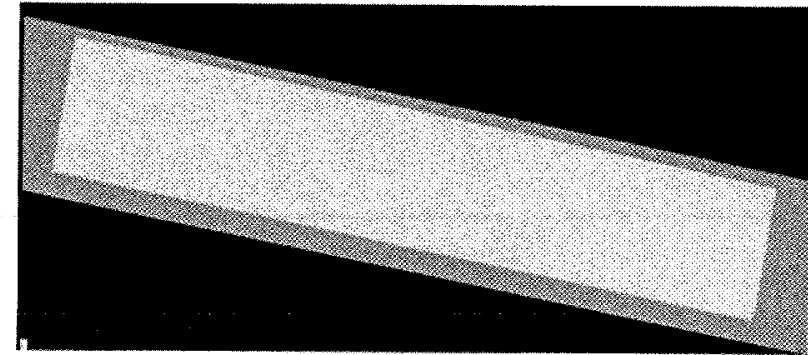

Figure 4. A portion of a typical Hyperion scene of Libya in WRS path 181 row 40 . The large highlighted rectangle represents the area used for comparisons.

One way to assess the uniformity of a test site is to find the standard deviation of the radiance or reflectance values across the site. However, the results of this assessment will also contain the variability in the instrument performing the measurements such as noise and flat fielding errors. This assessment is shown for the Railroad Valley in Figure 5 where the solid line shows the standard deviation of the average radiance reported by Hyperion and the dashed line shows the standard deviation of the average reflectance obtained with in situ measurements. Both the Hyperion imagery and in situ reflectance data were acquired on 13 July 2002. Since the Railroad Valley site represents only one pixel of MODIS, a 
standard deviation of MODIS data is not provided. The variability of the Libyan region is shown in Figure 6 as percent standard deviation of the radiance of the site where the solid line represents Hyperion on 4 June 2008 and the points represent Terra MODIS on 8 June 2008. The standard deviations shown here are representative of most clear days at this site. Except for in atmospheric absorption bands, the standard deviations of the uniformity of the site and instrument uniformity are found to be below $3 \%$ for both RRV and the Libyan Desert site, making them both good candidates for this study.

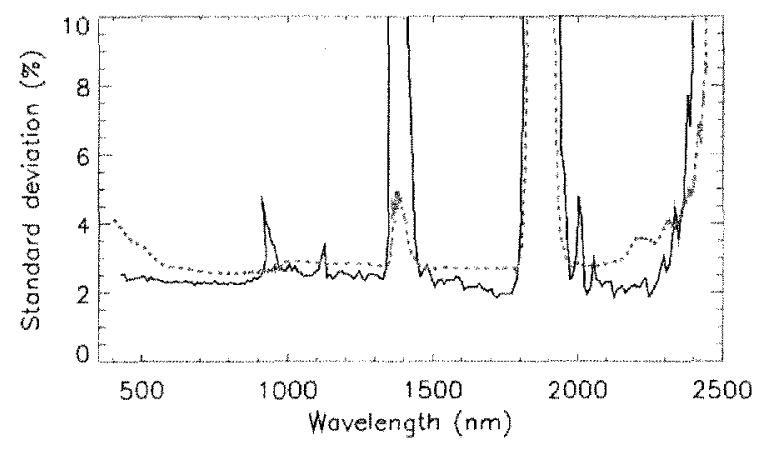

Figure 5. Indication of the RRV site variability. Percent standard deviations of the average radiance and reflectance measured by Hyperion (solid line) and in situ instruments (dashed line), respectively. Both data are from 13 July 2002.

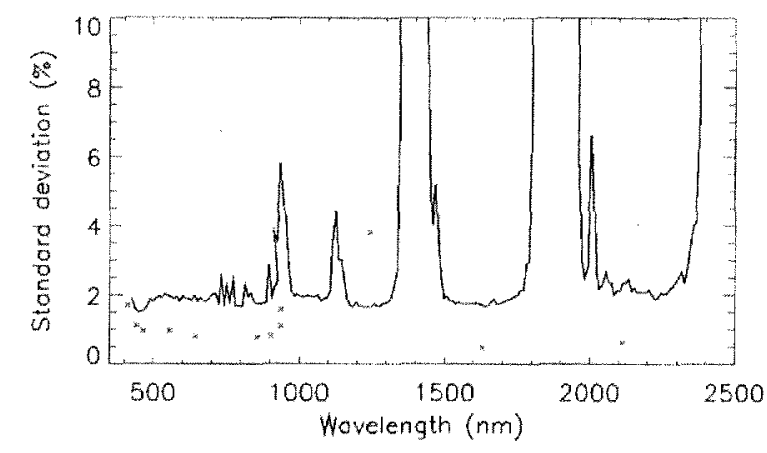

Figure 6. Indication of the Libyan site variability. Percent standard deviations of the average radiance measured by Hyperion (solid line) and Terra-MODIS (small markers). The dates of the data are 4 June 2008 and 8 June 2008 for Hyperion and MODIS, respectively.

\section{METHOD}

This work finds cross-calibration results for two scenarios that can essentially be labeled as coincident and noncoincident, referring to the image acquisition time and geometry. The coincident case, as well as results from the reflectance based approach, are held as a baseline for comparing the non-coincident case. Application of the noncoincident case is far more general since not nearly as many sensors fall under the coincident case.

The method used for both cases is nearly identical with the only difference being that the non-coincident case requires a search of image pairs from the two sensors that have matching geometries. Both coincident and non-coincident cases apply to the comparison between Hyperion and MODIS since they shared the same orbit with a 40-minute difference until mid-2005 at which time Hyperion deviated and only non-coincident comparisons are possible.

Imagery from both sensors is freely downloaded from the internet and radiance values from the Railroad Valley and Libyan tests sites are found. Hyperion level 1T data are obtained from the United States Geological Society (USGS) and Terra and Aqua MODIS calibrated radiance products MOD021 KM and MYD021KM, respectively, are obtained from National Aeronautical and Space Administration (NASA). Processing routines automate the procedure of defining the pixels that make up the test sites, finding averages and standard deviations of the radiance values, and recording any ancillary data such as view and solar geometry. The routine also applies a factor to correct of the difference in solar zenith angle.

For the non-coincident case, additional routines are developed that find Hyperion-MODIS image pairs that share similar view and solar geometry. Inputs to this routine are the library of images from both sensors, a maximum angular difference of the geometries between the two sensors, and the maximum temporal difference between the acquisition dates of the image pair. Restricting the geometry difference between an image pair reduces the uncertainties induced by the lack of knowledge about surface bidirectional functions and atmospheric effects. Restricting the temporal difference between the image pair may reduce the effect of differing atmospheric conditions. However, the more restriction placed on these parameters will result in fewer image pairs satisfying the geometry requirements. The results section will briefly explore the difference in results when the image pair matching restrictions are varied. 
A critical aspect of radiometric cross-calibration is accounting for spectral band differences of the sensors under study. For example, even though TM and ETM + have very similar spectral filters, it has been shown that their cross-calibration is affected by their spectral band differences at the $2 \%$ level $^{20}$. This work attempts to alleviate spectral effects by emulating MODIS bands with Hyperion bands. This allows for direct comparison of the two sensors. This is accomplished by band averaging the high spectral resolution data of Hyperion with the known relative spectral response functions of Aqua and Terra MODIS.

\section{RESULTS}

The results presented here consist of percent differences in radiance values of Hyperion and a reference with a functional form of

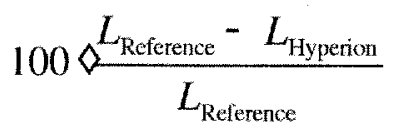

where $L_{R c f e r e n c e}$ and $L_{\text {Hyperion }}$ are the radiance values from the reference and Hyperion, respectively. The references employed in this work are at-sensor radiance values provided by the reflectance-based approach of vicarious calibration, solar-zenith corrected Terra-MODIS, and solar-zenith corrected Aqua-MODIS. When using a MODIS sensor for a reference, the Hyperion radiance is band averaged according to that sensor's relative spectral response. The MODIS bands used in this work are approximately centered at (units of nm): 442 (band 9), 466 (band 3), 553 (band 4), 645 (band 1), 856 (band 2), 904 (band 17), 1243 (band 5), 1632 (band 6), and 2119 (band 7).

The results compare cross-calibration scenarios that differ in image acquisition coincidence, test site used for the calibration, and reference sensor. Each plot is separated into visible-near-infrared (VNIR) and shortwave-infrared (SWIR) sections. Some of the scenarios will appear in more than one plot for comparison purposes, and in this case the plotting symbol for the scenario will remain consistent. All of the results are averages of multiple data points and therefore have some variance and this is displayed by the one standard deviation error bars in every plot.

The nearly ideal cross-calibration case of similar acquisition times with the same viewing geometry was possible for Hyperion and Terra MODIS image pairs before EO-1's orbit change in mid-2005. RSG performed the reflectance-based approach of vicarious calibration at Railroad Valley for Hyperion during this time. Results from both of these calibration methods are shown in Figure 7. RSG's ground-truth method provides hyperspectral radiance with is band averaged to the narrow spectral bands of Hyperion and since there are nearly 200 points, the data is plotted as a line. The sharp fluctuations in these results suggest that there is a spectral misregistration between the in situ measurements and Hyperion. The circles in Figure 7 represent differences between nearly coincident Terra-MODIS radiance and band averaged Hyperion radiance. The error bars on the symbols throughout this paper represent plus and minus one standard deviation of the averaged data. 